University of Rhode Island

DigitalCommons@URI

\title{
Readiness to Change as a Mediator of the Effect of a Brief Motivational Intervention on Posttreatment Alcohol-Related Consequences of Injured Emergency Department Hazardous Drinkers
}

\author{
L.A.R. Stein \\ University of Rhode Island, larstein@uri.edu \\ P. Allison Minugh \\ Richard Longabaugh \\ Philip Wirtz \\ Janette Baird \\ See next page for additional authors \\ Follow this and additional works at: https://digitalcommons.uri.edu/cprc_facpubs \\ Terms of Use \\ All rights reserved under copyright.
}

\section{Citation/Publisher Attribution}

Stein, L. A. R., Minugh, P. A., Longabaugh, R., Wirtz, P., Baird, J., Nirenberg, T. D.,...Gogineni, A. (2009). Readiness to Change as a Mediator of the Effect of a Brief Motivational Intervention on Posttreatment Alcohol-Related Consequences of Injured Emergency Department Hazardous Drinkers. Psychol. Addict. Behav., 23(2), 185-195. doi: 10.1037/a0015648

Available at: http://www.dx.doi.org/10.1037/a0015648

This Article is brought to you for free and open access by the Cancer Prevention Research Center at DigitalCommons@URI. It has been accepted for inclusion in Cancer Prevention Research Center Faculty Publications by an authorized administrator of DigitalCommons@URI. For more information, please contact digitalcommons-group@uri.edu. 


\section{Authors}

L.A.R. Stein, P. Allison Minugh, Richard Longabaugh, Philip Wirtz, Janette Baird, Ted D. Nirenberg, Robert F. Woolard, Kathy Carty, Christina Lee, Michael Mello, Bruce Becker, and Aruna Gogineni 


\title{
Readiness to Change as a Mediator of the Effect of a Brief Motivational Intervention on Posttreatment Alcohol-Related Consequences of Injured Emergency Department Hazardous
}

\section{Drinkers}

\author{
L. A. R. Stein, \\ Department of Psychology, Social Sciences Research Center, University of Rhode Island, Kingston; \\ Rhode Island Training School, Cranston, RI; and Center for Alcohol and Addiction Studies, \\ Department of Psychiatry and Human Behavior, Warren Alpert School of Medicine, Brown \\ University
}

\section{P. Allison Minugh,}

Datacorp, Providence, RI, and Center for Alcohol and Addiction Studies, Department of Psychiatry and Human Behavior, Warren Alpert School of Medicine, Brown University

\section{Richard Longabaugh,}

Center for Alcohol and Addiction Studies, Department of Psychiatry and Human Behavior, Warren Alpert School of Medicine, Brown University

\section{Philip Wirtz,}

Decision Sciences and Psychology, George Washington University

Janette Baird,

Lifespan, Providence, RI, and Department of Emergency Medicine, Warren Alpert School of Medicine, Brown University

\section{Ted D. Nirenberg,}

Lifespan, Providence, RI, and Departments of Emergency Medicine and Psychiatry and Human Behavior, Warren Alpert School of Medicine, Brown University

\section{Robert F. Woolard,}

Department of Emergency Medicine, Paul L. Foster School of Medicine, Texas Technical University Health Studies Center

\section{Kathy Carty,}

Vantage Point, Inc., West Warwick, RI

Christina Lee, Center for Alcohol and Addiction Studies, Department of Community Health, Warren Alpert School of Medicine, Brown University

Michael Mello, Lifespan, Providence, RI, and Department of Emergency Medicine, Warren Alpert School of Medicine, Brown University

Bruce Becker, and

\footnotetext{
(C) 2009 American Psychological Association

Correspondence concerning this article should be addressed to L. A. R. Stein, Department of Psychology, University of Rhode Island, Cancer Prevention Center, Kingston, RI 02881. LARStein@uri.edu.
} 
Lifespan, Providence, RI, and Department of Emergency Medicine, Warren Alpert School of Medicine, Brown University

\section{Aruna Gogineni}

Friends Research Institute (FRI), Baltimore, MD.

\section{Abstract}

Brief motivational interventions (BMIs) are usually effective for reducing alcohol use and consequences in primary care settings. We examined readiness to change drinking as a mediator of the effects of BMI on alcohol-related consequences. Participants were randomized into three conditions: (a) standard care plus assessment (SC), (b) SC plus BMI (BI), and (c) BI plus a booster session (BIB). At 12-month follow-up BIB patients had significantly reduced alcohol consequences more than had SC patients. Patients receiving BI or BIB maintained higher readiness scores 3 months after treatment than did patients receiving SC. However, readiness mediated treatment effects only for those highly motivated to change prior to the intervention but not for those with low preintervention motivation. BI and BIB for these patients decreased alcohol consequences in part because they enhanced and maintained readiness for those highly motivated prior to the intervention, but not for those with low motivation. Results are opposite of what would be expected from MI theory. An alternative explanation is offered as to why this finding occurred with this opportunistically recruited Emergency Department patient population.

\section{Keywords}

brief intervention; alcohol use; emergency medicine

Brief interventions in general (Bien, Miller, \& Tonigan, 1993), and brief motivational interventions (BMIs) in particular (Burke, Arkowitz, \& Dunn, 2002), have been shown to be effective for problem drinkers in reducing alcohol consumption and consequences in a variety of settings when compared with untreated controls. There is a great need in Emergency Departments (EDs) for effective treatments targeting alcohol use, because these settings have documented long-standing and high rates of admissions for minor and more serious alcoholrelated injuries (Cherpitel, 1992, 1996). BMIs adapted to the ED setting have been demonstrated to enhance alcohol-related outcomes (Blow et al., 2006; Longabaugh et al., 2001; Monti et al., 1999). However, not all studies of BMIs show positive results (e.g., Heather, Rollnick, Bell, \& Richmond, 1996; Hungerford et al., 1999; Richmond, Heather, Wodak, Kehoe, \& Webster, 1995). Therefore, it is important to examine the determinants of successful BMI interventions. As of yet, despite explicit theoretical formulation as to how and why motivational interventions (MIs) have their beneficial effects (Miller \& Rollnick, 1991, 2002), empirical research supporting these putative mechanisms of change has not yet fully materialized (Apodaca \& Longabaugh, in press; Burke et al., 2002; Dunn, Deroo, \& Rivara, 2001). The lack of identification of mechanisms of change for alcohol and substance abuse interventions is not unique to MI. Other treatments for alcohol use disorders also have scant evidence for the mechanisms by which they are effective (Longabaugh \& Wirtz, 2001;

Morgenstern \& Longabaugh, 2000). More generally, the search for mechanisms of change in behavioral treatments of most disorders has been found wanting (Kazdin \& Nock, 2003; Nock $\&$ Kazdin, 2005). Thus, identification of the mechanisms of change operative in behavioral interventions has become a high priority for the treatment research field more generally (Kazdin $\&$ Nock, 2003) and for alcohol-specific interventions in particular (Willenbring, 2007). When such mechanisms are identified, it will become more feasible to successfully disseminate efficacious treatments to everyday clinical settings. 


\section{Readiness to Change as a Mechanism}

The present study is a planned secondary analysis of the role of readiness to change drinking as a mediator of the effects of a BMI on the posttreatment alcohol-related consequences of opportunistically identified subcritically injured hazardous drinkers presenting for treatment of their injuries in an ED. Readiness to change was chosen as the mechanism of interest because it is central to MI theory (Hettema, Steele, \& Miller, 2005). As is described in more detail by Longabaugh et al. (2001), patients identified as hazardous drinkers were randomized into one of three intervention groups: standard ED care (SC); SC plus a BMI while the patient is in the $\mathrm{ED}$ (BI); and BI plus a single booster session conducted 1 week (on average) after the original ED visit (BIB). Patients were reassessed on outcomes twice (3 months and 1 year) after the criterion ED visit. Longabaugh et al. (2001) found that BIB decreased 1-year alcohol-related negative consequences more than did SC $(p<.001)$ and that BI outcomes were intermediate between these two groups (but significantly different from neither). The present study explores readiness to change as a putative mediator of this treatment—outcome relationship.

Readiness to change (as part of the Transtheoretical Model; Prochaska \& DiClemente, 1983) has been criticized for being based on discrete stages, for being poorly measured, and for being treated as an outcome of behavior change rather than as a proxy outcome that is secondary to actual behavior change (Bridle et al., 2005). We note that it is a central construct in MI theory (Hettema et al., 2005) and that other investigators have found this construct to be empirically meaningful (Rohsenow et al., 2004; Slavet et al., 2005; Stotts, Schmitz, Rhoades, \& Grabowski, 2001; Velicer, Redding, Xiaowu, \& Prochaska, 2007). As is described below, we utilized a validated measure to assess readiness to change, and this study views the readiness construct as a measure secondary to behavioral outcome.

\section{Pathways by Which Readiness May Produce Effects}

Specifically, the a priori planned analysis tests first the hypothesis that BMI-based interventions will enhance and maintain the patient's motivation to change their drinking (measured at 3 months postintervention) more than will SC. If this is not the case, then the putative mechanism of change through which BMI operates on negative consequences would be severely challenged. Second, it is hypothesized that change in motivation (baseline to 3 months posttreatment) is negatively associated with change in negative consequences (baseline to 12-months posttreatment) and that this is independent of any relationship between treatment and change in negative consequences. Collectively, these two hypotheses represent a putative causal pathway in which change in motivation mediates the relationship between BMI treatment and change in negative consequences.

Lastly, because the second part of the hypothesized causal chain was not supported, we examine the extent to which this part of this hypothesized causal chain (viz., the independent relationship between change in motivation and change in negative consequences) is moderated by baseline readiness to change. The underlying thesis is that the relationship between motivational change and change in negative consequences may be affected by baseline readiness to change in either of two contrasting ways. MI theory (Miller \& Rollnick, 1991,2002) posits that patients who are less motivated initially will be more responsive to an intervention focused on increasing and maintaining motivation to change substance use: Under this model, the relationship between change in motivation and change in negative consequences would be expected to be strongest among those whose initial motivation level was low. This is the obvious derivation from MI theory, and in fact there are two studies that have found indirect support for this model. Two studies (Rohsenow et al., 2004;Stotts et al., 2001), both conducted with treatment-seeking populations, reported that baseline readiness to change moderated the MI treatment effect such that those less ready to change prior to the intervention benefited more than did the comparison 
group (while those more ready to change benefited less than did the comparison group). Neither of the above cited studies reported testing whether it is the change from pre-intervention to postintervention level of readiness that accounted for the differential effects of MI on more and less motivated patients.

While MI theory would seem particularly applicable to patients presenting for treatment at an alcohol or addiction specialty clinic, this is less clearly applicable to patients presenting at an ED for treatment of an injury that may or may not be alcohol related. Among such patients (opportunistically identified as harmful drinkers), for those who are not already motivated to reduce their drinking, a brief MI intervention may not be sufficient to lead to sustained behavioral changes that reduce their alcohol-related negative consequences from drinking. Rather it may be the case that only patients already motivated to reduce their alcohol consumption will benefit from an intervention focused on this goal. Under this model, the relationship between change in motivation and change in negative consequences would be expected to be strongest among those whose initial motivation level was high.

Both models generate the expectation that baseline motivation will moderate the relationship between change in motivation and change in negative consequences, although the two models predict contrasting directions for this moderational effect. As a result, this hypothesis is tested nondirectionally.

While several studies have failed to find that baseline motivation moderates treatment's effects on outcome (e.g., DiClemente, Marinilli, Singh, \& Bellino, 2001; Emmen, Schippers,

Wollersheim, \& Bleijenberg, 2005; Miller, Wilbourne, \& Hettema, 2003; Monti et al., 1999), the aim of this analysis differs in that we test whether pretreatment readiness to change has an effect on the extent to which increased readiness to change resulting from the BMI mediates the effect of the intervention on outcome.

In summary, the aims of the a priori analysis are as follows: (a) to test whether BMI (in comparison with SC) increases or maintains readiness to change drinking 3 months after the intervention and (b) to test whether this increased readiness to change 3 months postintervention mediates the effectiveness of $\mathrm{BMI}$ vs. SC in reducing negative consequences from drinking 12 months postintervention.

The aim of the post hoc analysis is to test whether the pre-intervention level of readiness to change moderates the mediating effect of 3-month posttreatment readiness to change on the relationship of intervention condition to 1-year drinking outcome.

\section{Method}

\section{Patient Population}

The treatment setting was an urban, Level 1 ED trauma center located in southern New England. In order to qualify for study inclusion, consenting patients had to be at least 18 years old, present to the ED with an injury that did not result in admission to the hospital, and receive a hazardous drinking assessment. Hazardous drinking was identified by any of three criteria: (a) a positive test for alcohol as measured by breathalyzer (BAC $\geq 0.003 \mathrm{mg} / \mathrm{dl}$ ) while in the ED, (b) a selfreport of ingesting alcohol within $6 \mathrm{hr}$ prior to the injury, ${ }^{1}$ or (c) a score of 8 or more on the Alcohol Use Disorders Identification Test (AUDIT).

Patients were excluded from the study if (a) they spoke neither English nor Spanish, (b) they lived more than $1 \mathrm{hr}$ distance from the hospital, (c) they had no identifiable residence, (d) they

\footnotetext{
${ }^{1}$ Only 41 patients drank within $6 \mathrm{hr}$ of their injury, who were not also either BAL positive or $\geq 8$ on the AUDIT.
} 
were under arrest at the time of the ED visit, (e) they were judged by the ED staff as having a psychiatric disorder, or (f) they had been previously diagnosed with alcohol dependence or abuse. Participation was delayed for all patients until they were able to give informed consent. We judged patients as able to give consent when their BAC was $\leq .20 \mathrm{mg} / \mathrm{dl}$, their cognition was unimpaired as determined by a brief mental status examination, and they were able to articulate comprehension of the study and what they were being asked to do as study participants. Patients were required to have a BAC of $\leq .10 \mathrm{mg} / \mathrm{dl}$ prior to initiating the intervention.

The research staff identified 3756 injured patients who were injured but not hospitalized during the sampling period. Of these, $382(10 \%)$ indicated that they did not wish to participate in the study. Because of the sequence of the study enrollment procedure, we cannot tell what percentage of these 382 persons would have met all eligibility criteria. However, if all of these 382 persons had also been eligible, then the refusal rate would have been $41 \%$ ( 539 [see below] $+382=921 ; 382 / 921=0.41)$.

Of the patients who met the study inclusion criteria and agreed to participate $(N=539), 78 \%$ were men, and the average age was 27 years (standard deviation, $S D,=9$ ). The ethnic/racial composition of the study sample was $72 \%$ White, $14 \%$ Latino/Hispanic, $10 \%$ African American, $<1 \%$ Asian, and $<1 \%$ Native American, and 3\% identified themselves as belonging to other ethnicities or races. Most patients (77\%) reported their marital status as single, and most (72\%) were employed. The average participant's baseline AUDIT score was 12.8; the average Drinker's Inventory of Lifetime Consequences (DrInC) score was 15.6. The study sample was representative of the population that utilizes this ED. After excluding participants for missing either 3-month or 12-month data, the number of participants in the final sample was $N=417(77 \%)$. There were no statistically significant differences between those with missing data and those included in the final sample on important baseline characteristics (AUDIT, DrInC, readiness to change, treatment assignment, ethnicity, age, education, marital status, employment, and gender).

\section{Recruitment Procedures}

When interventionists were on duty, they reviewed patient admission records for potentially eligible patients. The interventionist approached injured patients and asked permission to screen them and to explain the study briefly. The interventionist administered the AUDIT to patients who agreed. These patients also answered questions on a brief mental status exam. The interventionist also asked these patients if they had consumed alcohol within $6 \mathrm{hr}$ of their injury. If they met these criteria, the interventionist then asked for informed consent to participate in the research study. We then asked patients to take a breath analyzer test to determine whether their BAC was within the predetermined limits for completing the assessment instruments.

Patients who met all eligibility requirements received a written consent form that they either read or had read to them, and then were queried regarding key aspects of their participation. Each participant received $\$ 15$ for completing the baseline instruments. Prior to intervention assignment, the patient was retested to ensure that $\mathrm{BAC}$ was $\leq .10 \mathrm{mg} / \mathrm{dl}$ prior to initiation of the intervention. The university and hospital institutional review boards approved all study procedures.

\section{Assessment}

An interventionist administered an assessment battery to study participants prior to randomly assigning them to treatment condition. Pertinent to the present study, the baseline assessment included the AUDIT, the DrInC, and a decision ladder regarding their readiness to change their 
drinking. In addition to these instruments, we administered other instruments that helped characterize the study patients, the circumstances surrounding their injuries and information necessary to locate them for follow-up interviews. The complete assessment battery averaged 30 to $40 \mathrm{~min}$. Independent research assistants who were uninformed as to the patient's treatment assignment collected the 3-mont and 12-month follow-up assessment data.

Measures

AUDIT-This is a 10-item self-report measure that screens for hazardous or harmful drinking (Saunders, Aasland, Babor, de la Fuenta, \& Grant, 1993). Items include alcohol consumption quantity and frequency measures, heavy drinking, dependence symptoms, tolerance, and alcohol-related negative consequences. The AUDIT's internal consistency alpha is .80 , and it reliably distinguishes between patients with hazardous and harmful drinking histories and those without such histories (Fleming, Barry, \& MacDonald, 1991; Saunders et al., 1993). An AUDIT cut-off score of $\geq 8$ produces $85 \%$ sensitivity and $89 \%$ specificity for hazardous or harmful drinking (Cherpitel, 1995). The measurement window was the past year.

DrInC-As used in the present study, the DrInC is a 45-item self-report questionnaire that asks about negative consequences experienced from drinking. The DrInC was validated on an alcohol treatment - seeking population of 1728 inpatients and outpatients (Miller, Tonigan, \& Longabaugh, 1995). It has well-established psychometric properties (Miller et al., 1995). As validated in Project MATCH, the DrInC provides a measure of negative consequences from drinking that is sensitive to change and correlates with other outcome measures (e.g., percentage of drinking days, average number of drinks on a drinking day, subjective wellbeing, and psychosocial functioning; Cisler \& Zweben, 1999).

We administered the lifetime version of the DrInC at baseline. At this time, we asked the patient whether he or she had ever experienced any of the consequences listed on the measure. At 1year follow-up, we asked patients to recall how often they had experienced any of the consequences in the past year $(0=$ never to $3=$ daily or almost daily $)$. The total DrInC score at baseline served as the covariate, and the total score at the 1-year follow-up point served as the dependent variable indicative of clinical out-come. The average score on the lifetime DrInC is comparable to the lowest decile for a treatment-seeking population, as is the average DrInC score at follow-up.

Two other instruments measuring drinking outcomes are potentially relevant to the present study. The AUDIT, which includes an item measuring heavy drinking days, was also administered at follow-up data points, as was the Injury Behavior Checklist (IBC). As is reported in the original article (Longabaugh et al., 2001), treatment groups did not differ on heavy drinking days (Longabaugh et al., 2001, p. 812). Moreover, reduction in drinking in itself was not the primary aim of the intervention (Longabaugh et al., 2001, pp. 806, 810). Rather, the aim of the intervention for this opportunistically recruited sample was reduction of negative consequences from drinking. For both of these reasons, heavy drinking days were not considered as a target behavior for mediation of treatment/control outcome differences.

The IBC was not considered as a dependent variable in the present study because treatment's effect on reported injury reduction was only marginally significant in the comparison of BIB and SC ( $p \leq .04$, one-tailed test). Because the focus of the present study was on studying motivational readiness as a mediator of the BIB versus SC difference, we chose to focus on the one primary dependent variable that was unquestionably affected by treatment condition.

Readiness to change-Motivation, or readiness to make a change in drinking behavior, was measured using an adaptation from Beiner and Abrams' (1991) Readiness to Change Contemplation Ladder. The adaptation was validated on an ED treatment-seeking population 
of injured drinkers (Longabaugh et al., 1995). The patient is asked to place his or her readiness to change on a rung of a contemplation ladder with response categories ranging from (0) no thought of changing to (10) taking action to change (i.e., cutting down). The Readiness to Change drinking ladder was grouped conceptually into the following rungs: (0) No thought of changing $(N=171)$; (1-3) Think I need to consider changing someday $(N=99)$; (4-6) Think I should change but not quite ready $(N=85)$; (7-9) Starting to think about how to change my drinking patterns $(N=103)$; and (10) Taking action to change, such as cutting down $(N=79)$. These groupings are consistent with Precontemplation, Contemplation, Determination, Preparation, and Action in Prochaska and DiClemente's (1983) Stages of Change.

\section{Treatment Assignment}

Once patients completed the baseline assessment battery, the interventionist opened an envelope that contained the patient's treatment condition assignment. Patients were randomly assigned to one of three treatment conditions: standard care (SC), brief intervention (BI), or brief intervention plus booster (BIB). If the patient's treatment assignment was SC, then the interventionist simply scheduled the follow-up interview with the patient, and the patient was discharged from the hospital following standard medical practice. Irrespective of treatment condition assignment, patients who exhibited a need for alcohol treatment received a referral list of treatment services. When patients were assigned to a treatment condition (either BI or BIB), the intervention then began. After the intervention, the interventionist opened a second sealed envelope, which revealed whether or not the patient would receive the booster condition (BIB). If a participant's treatment assignment was to the single session intervention (BI), the interventionist scheduled the patient's follow-up interview, and standard hospital discharge was followed for the patient's release from the ED. If a participant's treatment assignment was to the BIB condition, the interventionist scheduled the booster session. Booster sessions took place within 7 to 10 days of the initial ED visit. Prior to standard discharge, the interventionist scheduled the follow-up research interview with these patients as well.

\section{Interventions}

SC-Patients assigned to SC received the same customary care they would normally receive in the hospital ED in the absence of the research protocol. For details, see Longabaugh et al. (2001).

$\mathrm{BI}-\mathrm{BI}$ occurred after the baseline assessment was completed and prior to the patient being discharged from the hospital. The session lasted 40-60 min. The intervention was based on motivational enhancement treatment implemented in Project MATCH (Miller, Zweben, DiClemente, \& Rychtarik, 1992). The MATCH motivational enhancement manual was adapted for the ED setting (Nirenberg, Sparadeo, \& Longabaugh, 1996). The underlying principles are those of motivational interviewing (MI; Miller \& Rollnick, 1991, 2002), an approach that uses accurate empathy, warmth, and genuineness and in which confrontation is proscribed. The aim of the intervention was to reduce or eliminate negative consequences for drinking, especially alcohol-related negative consequences. As such, the initial MI session began with open-ended questions about the patient's injury and a possible connection between the injury and alcohol use. If the patient assessed that there was no connection, the interventionist broadened the focus to include negative effects the patient had identified on the DrInC as attributable to drinking. Patients were assisted in determining whether or not they wanted to change any behaviors and, if so, which behavior they wished to target. For the target behavior a decisional balance form indicating the pros and cons of change was completed. Finally, the patient completed a change plan for the targeted behavior (Longabaugh et al., 2001, p. 810). 
BIB-Patients assigned to the BIB condition had a return appointment scheduled within 7 to 10 days of the initial session. This follow-up session took place at the hospital. The principles of MI also formed the basis for the follow-up intervention. For further details regarding the initial and booster session interventions, see Longabaugh et al. (2001).

\section{Therapist Training and Monitoring}

Interventionists-The clinical supervisor trained eight interventionists to conduct the baseline recruitment, assessment, and BMI. Interventionists were experienced clinicians who previously worked in both research and counseling settings.

Training and monitoring fidelity to treatment-Counselor training included relevant reading materials (Miller et al., 1992,1995), didactic sessions and discussion, role-playing practice, and observing supervisors conduct of the intervention, and when appropriate, volunteer patients participated in the presence of the supervisor. When each trainee demonstrated mastery of the techniques, the supervisors certified the clinician to conduct the intervention with study patients. Counselors met weekly in a group with their clinical supervisor. In addition to the group sessions, clinical supervisors met one-on-one with the interventionists on an as-needed basis. Audiotaped sessions were also used as part of supervision. Both patients (Lee et al., 2007) and interventionists (Baird et al., 2007) completed postsession evaluation forms that provided information on the extent to which the interventionist used MI principles and established a helpful alliance with the patient. For more details regarding interventionist qualifications, training and monitoring, see Longabaugh et al. (2001).

\section{Follow-Up Assessment}

A proactive, rigorous procedure was used for the conduct of the patients' follow-up interviews (Woolard et al., 2004). Patients were asked to return to the hospital for follow-up interview visits. Those unwilling or unable to do so were offered the option of completing the follow-up questionnaire at an off-site location or by a combination of telephone and mail. Patients were compensated $\$ 25$ for each completed interview. At 1-year follow-up, 417 (77.4\%) of the enrolled patients who provided the necessary 3-month follow-up data completed the assessment.

\section{Analytical Procedure}

All analyses were conducted using Version 9.1.3 (SP4) of SAS (SAS Institute, Inc., Cary, NC). Parameter estimates and standard errors for both the mediation model and the moderated mediation model were generated using standard ordinary least squares (OLS) regression. Change in motivation was implemented by covarying on baseline motivation, and change in drinking consequences was implemented by covarying on baseline drinking consequences. In order to minimize the effect of skewness on the parameter estimates, the square root transformation was applied to baseline negative consequences, and the log transformation was applied to 12-month negative consequences.

Two approaches were used for testing the mediation and the moderated mediation models. The classical multistep approach proposed by Baron and Kenny (1986) has lower power in the nondirectional case but correctly handles directional alternative hypotheses of the two mediational subpaths (as are proposed here). The product of coefficients approach (MacKinnon, 2008) is more powerful (MacKinnon, Fairchild, \& Fritz, 2007) but does not adequately handle directional alternative hypotheses of the two mediational subpaths. Edwards and Lambert (2007) provided an alternative (path analytic) approach for estimating models that integrates mediation and moderation; however, the comparative statistical power of this approach remains unclear (see also Muller, Judd, \& Yzerbyt, 2005; Preacher, Rucker, \& Hayes, 
2007). The standard error of the product of coefficients was estimated using the PRODCLIN2 program (MacKinnon, Fritz, Williams, \& Lockwood, 2007). This program is available for download (see http://www.public.asu.edu/ davidpm/ripl/Prodclin/). In the moderated mediation analysis we have focused more narrowly on BIB versus SC, because Longabaugh et al. (2001) found treatment effects for this comparison and not for BI versus SC, and because theoretically, BIB should enhance motivation more than should BI.

\section{Results}

On the 12-month negative consequences measure, the mean score was 17.2 ( $S D=19.5$; range $=0-135$ ), which is comparable to normative 12-month scores for the DrInC (Miller et al., 1995). Distributional characteristics for baseline and 3-month motivation scores, and for baseline and 12-month DrInC scores, are shown in Table 1.

As is shown in Table 2, patients in the BIB and BI conditions reflected a higher average 3month motivation level than did patients in SC. After adjusting for baseline motivation level, this difference was statistically significant for $\mathrm{BIB}, t(413)=1.72, p \leq .05$, as was the average $\mathrm{BIB}$ and BI treatment effect when compared with SC, $t(413)=1.76, p \leq .04$.

Participants in the BIB and BI conditions reflected a lower severity of posttreatment negative consequences than did participants in the SC. After adjusting for baseline negative consequences, this difference was statistically significant for BIB, $t(423)=-2.59, p \leq .01$, as was the average $\mathrm{BIB}$ and $\mathrm{BI}$ treatment effect when compared with $\mathrm{SC}, t(423)=-2.36, p \leq .01$.

\section{Mediation Model}

These results are suggestive of, and provide the impetus for a formal test of, a model in which change in motivation mediates the relationship between treatment and change in negative consequences. The classical approach to mediation, based on the multistep approach presented by Baron and Kenny (1986), is comparatively weak in power when the mediational model is nondirectional. In contrast, the product of coefficients approach (e.g., MacKinnon, 2008) provides for considerably greater power in a nondirectional context but does not provide for an adequate test of the directionality of specific components in the putative causal chain. Because the two approaches complement each other, we used them both in this analysis.

Baron and Kenny's (1986) first condition requires that the treatments differ in their effect on the outcome variable. Consistent with our directional hypothesis, participants randomly allocated to the BIB treatment reflected (on average) significantly fewer negative consequences 12 months posttreatment than did those participants who were allocated to the SC treatment, $t$ $(413)=-2.70, p \leq .004$ (see Table 3). This corresponds to link $\mathrm{c}$ in Figure 1.

Baron and Kenny's (1986) second condition stipulates that the treatments must differ in their effects on the putative mediator. Again, consistent with our directional hypothesis, participants in the BIB treatment reflected (on average) significantly greater change in motivation between baseline and 3 months than did participants in the SC treatment, $t(413)=1.72, p \leq .04$. This corresponds to link $a$ in Figure 1.

The third statistical condition of Baron and Kenny (1986) stipulates that the putative mediator must be related to the outcome, independent of any effects of treatment. We found that, after controlling for treatment, baseline motivation, and negative consequences of drinking, higher motivation was associated with greater negative consequences at 12-month follow-up (albeit nonsignificant), $t(411)=1.63, p>.05$, which was inconsistent with our directional expectation. This corresponds to link $b$ in Figure 1. 
Given that a product of coefficients approach provides a more powerful test of mediation in the absence of directional alternative hypotheses, we were interested in knowing whether mediation was occurring in a direction contrary to our hypothesized effect. The product of coefficients test was found to be nondirectionally nonsignificant because zero was contained inside the 95\% confidence interval for the product $(\hat{a}=.29, S E(\hat{a})=.17, \widehat{b}=.06, S E(\widehat{b})=.04$, $95 \% \operatorname{LCL}(\widehat{a} \widehat{b})=-.004,95 \% \operatorname{UCL}(\widehat{a} \widehat{b})=0.49)$. Note that $S E=$ standard error, LCL $=$ lower confidence limit, $\mathrm{UCL}=$ upper confidence limit, $\hat{a}=$ is the link between the independent variable of interest and the putative mediator, and $\widehat{b}=$ is the link between the putative mediator and the dependent variable, after controlling for the independent variable (see MacKinnon, 2008).

\section{Moderated Mediation Model}

One explanation for the lack of a fully mediated model lies in the possibility that link $b$ in Figure 1 differs by level of pretreatment motivation; this is equivalent to Model 3 explicated by Preacher et al. (2007). In order to test this "moderated mediation model," we used two alternative approaches: one specified by Muller et al. (2005), and the other specified by MacKinnon (2008).

Muller et al. (2005) specified four criteria for testing moderated mediation. First, the independent variable (treatment condition) must be significantly related (in the hypothesized direction) to the change in negative consequences. Second, the magnitude of this effect should not depend on the putative moderator (baseline motivation). Third, the effect of the putative mediator (change in motivation) on change in negative consequences should be related to the putative moderator (baseline motivation). Fourth, there should be an overall effect of the independent variable (treatment condition) on the putative mediator (change in motivation) in the hypothesized direction. ${ }^{2}$

The four conditions specified by Muller et al. (2005) were all met. First, 12-month negative consequences was significantly lower in the BIB condition than in the SC condition after controlling for baseline negative consequences, $t=-2.70, d f=413, p \leq .0036$. Second, after controlling for baseline negative consequences, the effect of treatment condition on 12-month negative consequences was not found to differ significantly across different levels of baseline motivation, $F(2,416)=0.88, p>.05$. Third, the Baseline Motivation $\times 3$-Month Motivation interaction was found to be statistically significant, $t(416)=-3.70, p \leq .0002$. Fourth, after controlling for baseline motivation, 3-month motivation was significantly higher in the BIB condition than in the SC condition, $t(413)=1.72, p \leq .04$.

MacKinnon's (2008) approach to moderated mediation expands on the Muller et al. (2005) approach by identifying the levels of the putative moderator at which the mediation occurs. Following MacKinnon (2008), we tested the mediation model described above at each of the five levels of baseline motivation. As is reflected in Table 3, these analyses revealed that the unexpected positive relationship between change in motivation and subsequent change in negative consequences was confined to those participants reporting low levels of pretreatment motivation. Among those participants reporting high levels of pretreatment motivation, greater reduction in motivation was associated with greater subsequent increase in negative consequences, as was initially hypothesized. The results presented in Table 3 also show that motivation significantly mediates the relationship between treatment and negative consequences for those reporting high levels of pretreatment motivation (the interval defined

\footnotetext{
${ }^{2}$ The criteria specified here correspond to moderation of the link between the putative mediator and the outcome variable. Muller et al. (2005) presented a parallel, but slightly different, set of criteria for models in which the link between the independent variable and the putative mediator is moderated.
} 
by the lower and upper bounds of a $95 \%$ asymmetric confidence interval for $\mathrm{a} * \mathrm{~b}$ was not found to include zero) but not for those reporting lower levels of pretreatment motivation. The magnitude of this mediational effect is estimated to be approximately $30 \%$ (i.e., $\widehat{a} \widehat{b} / \widehat{c} * 100$ ).

\section{Discussion}

As was expected, the combined group of BIB and BI produced significantly fewer alcoholrelated negative consequences 1 year after the criterion $\mathrm{ED}$ visit than did the $\mathrm{SC}$ group. Consistent with a dose/response effect, BIB alone was significantly more effective than was SC, while BI alone was directionally more effective than was SC but not significantly so. Consistent with the hypothesis that a mechanism through which this superior outcome might be achieved is enhanced motivation, we found that BIB and BI combined maximized readiness to change from pretreatment to 3 months posttreatment more than did SC. Again, consistent with a dose/response effect, the difference between BIB and SC was significant, whereas the difference between $\mathrm{BI}$ and $\mathrm{SC}$ was not significant. When pretreatment readiness to change was taken into account as a moderator of the strength of the motivation/outcome relationship, we found that the relationship between 3-month motivation and 12-month negative consequences became significantly more negative at higher levels of pretreatment motivation.

The direction of this interaction is opposite what would be hypothesized from a simple derivation of MI theory, that MI works in part through increasing the motivation of those less motivated for change. The simple hypothesis would have been that those low in readiness to change would benefit from an MI-based intervention more than those already high in readiness to change. The results obtained here suggest a more nuanced hypothesis: That two sessions of BMI will be sufficient to sustain the motivation to change for those more highly motivated to change prior to the intervention, but for those less ready to change prior to the intervention, two sessions of BMI are insufficient to motivate the patient to mobilize his or her resources to initiate or sustain the targeted behavioral change. Further research is necessary to test this hypothesis and replicate our finding. In this context, it should be remembered that those targeted for an intervention directed at achieving a reduction in alcohol-related negative consequences were opportunistically identified. They did not present in the ED seeking treatment for hazardous drinking but rather for their injury. Thus, we speculate that a BMI of the intensity we offered in this context is not robust enough to fully engage and mobilize patients unready for change following a subcritical injury that might or might not be perceived as drinking related. An independent analysis of this data set failed to find a hypothesized increase in injury attribution to self or alcohol as a mediator of the BIB-outcome relationship (Carty, 2005). Thus, we are left with the unanswered question as to why BMI is not effective with these patients who are not seeking to change their alcohol use prior to the BMI intervention.

Given these differential effects for patients high and low in readiness to change prior to the intervention, a very important question to address is, "What are the predictors of pretreatment readiness to change drinking?" Minugh et al. (2009) have investigated the question with this ED population. They find that one of the most important predictors of pretreatment readiness to change drinking is a history of self-reported alcohol-related negative consequences. This supports the notion that there must be some minimum threshold of recognition of alcohol consequences present for a BMI to have a sustaining effect.

In the absence of a more effective intervention for those not ready to change, there is a strong implication that ED patients be screened for readiness to change prior to undertaking a BMI intervention. This will permit a more efficient use of available treatment resources. In our study, up to $50 \%$ of patients who might meet study criteria refused research enrollment. This suggests that perhaps only a quarter or so of patients meeting our criteria for hazardous drinking would 
benefit from the BMI intervention we implemented. Systematic screening of this population could result in a much more cost-effective intervention.

There also remains the very important question as to why more, rather than less, motivated patients would benefit from this BMI intervention. Lee et al. (2009), independently analyzing this same data set, have focused on patients receiving the BMI (either BI or BIB) intervention. They have found that patients who complete a higher-quality change plan aimed at reducing the negative consequences from alcohol consumption show a greater reduction in alcoholrelated consequences than do BMI patients who fail to do so. Lee et al. also found that patients with higher readiness to change prior to the BMI are more likely to complete high-quality plans involving reduction of consequences from alcohol reduction, but that pretreatment readiness does not in itself predict 12-month alcohol-related consequences.

Putting all of these pieces together, the hypothesized causal chain is this: (a) patients with significant alcohol-related consequences prior to the intervention are more ready to reduce their drinking (Minugh et al., 2009). (b) Of these, patients who are assigned to MI are more likely to develop a change plan for reducing negative consequences from alcohol consumption (Lee et al., 2009). (c) Those who receive two sessions of MI are more likely to complete a quality change plan than are those who receive only one MI session (Baird et al., 2007). (d) Those with a quality change plan are more likely to sustain high motivation to reduce alcoholrelated consequences through at least 3 months following the intervention and (e) are more likely to have reduced their negative consequences through 12 months after the intervention (Lee et al., 2009).

The complexity of these findings have a very important implication for research seeking to identify mechanisms of change in behavioral interventions. As is summarized at the beginning of this article, the results of these efforts to date have been meager. It has been suggested elsewhere (Longabaugh, 2007; Longabaugh \& Wirtz, 2001) that this paucity of return may be attributable to underspecification of the theoretical formulation to account for the complexities of research findings. In the present instance, we posited a single mediator, readiness to change drinking, as the mechanism that would account for the effect of the BMI intervention on outcome. Our results have shown us that this single variable was not sufficient to account for the observed treatment-outcome relationship. It was only when we took into account pretreatment level of readiness to change, and added it to the conceptual model, that we were able to reach at least a partial understanding of the role of readiness to change in mediating the treatment effect. This understanding required recognition of the importance of pretreatment motivation, as well as its interaction with change in motivation following treatment, as well as the investigation of preceding and subsequent variables in the hypothesized causal chain. It is our expectation that more complex causal chains that may include moderator-mediator interactions will be necessary to develop theoretical models that more closely approximate the dynamics of the treatment effects that we are attempting to explain.

The present research has several limitations. Findings are limited to opportunistically identified hazardous drinkers in an ED setting. For patients seeking treatment for their alcohol problems, pretreatment motivation to reduce their drinking may already be present. If all such patients are already ready to change, their preintervention motivation may not be a likely moderator of their treatment success.

Another limitation may have to do with measurements. We used a brief measure of readiness to change drinking to index both pretreatment motivation and change in motivation. It may be that a more comprehensive measure would have yielded different results. Despite this limitation, however, we did find evidence that the measure used was theoretically coherent in the context of our results. 
Another limitation is that readiness to change drinking was not measured for all groups until 3 months after the intervention. To address the question of temporal order (e.g., whether the change in drinking consequences preceded the change in motivation or vice versa; Kazdin \& Nock, 2003), it would be optimal if all groups had been assessed for readiness to change immediately following their ED experience. However, this shortcoming is mitigated somewhat by the fact that readiness to change drinking was reassessed 9 months prior to alcohol-related consequences, which in turn was measured 1 year after the intervention.

Finally, as is usually the case, these findings need to be replicated in similar and dissimilar samples before we can be assured that these results are reliable and the extent to which they are generalizable across different populations of substance abusers and treatment settings. Most specifically, these results have been obtained with a BMI. It may be the case that the same results would be obtained with other brief interventions as well.

\section{Acknowledgments}

This study was funded by a grant from NIAAA (No. 5R01AA09835) to Richard Longabaugh, principal investigator.

\section{References}

Apodaca TR, Longabaugh R. Mechanisms of action in motivational interviewing: A review of the evidence. Addiction 2009;104:705-715. [PubMed: 19413785]

Baird J, Longabaugh R, Lee CS, Nirenberg TD, Woolard R, Mello MJ, et al. Treatment completion in a brief motivational intervention in the emergency department: The effect of multiple interventions on therapist's behavior. Alcoholism: Clinical and Experimental Research 2007;31(S3):71S-75S.

Baron RM, Kenny DA. The moderator-mediator variable distinction in social psychological research: Conceptual, strategic, and statistical considerations. Journal of Personality and Social Psychology 1986;51:1173-1182. [PubMed: 3806354]

Beiner L, Abrams DB. The contemplation ladder: Validation of a measure of readiness to consider smoking cessation. Health Psychology 1991;10:360-365. [PubMed: 1935872]

Bien TH, Miller WR, Tonigan JS. Brief interventions for alcohol problems: A review. Addiction 1993;88:315-335. [PubMed: 8461850]

Blow FC, Barry KL, Walton MA, Maio RF, Chermack ST, Bingham CR, et al. The efficacy of two brief intervention strategies among injured, at-risk drinkers in the emergency department: Impact of tailored messaging and brief advice. Journal of Studies on Alcohol 2006;67:568-578. [PubMed: 16736077]

Bridle C, Riemsma R, Pattenden J, Sowden A, Mather L, Watt I, et al. Systematic review of the effectiveness of health behavior interventions based on the transtheoretical model. Psychology and Health 2005;20:283-301.

Burke, BL.; Arkowitz, H.; Dunn, C. The efficacy of motivational interviewing and its adaptations: What we know so far. In: Miller, W.; Rollnick, S., editors. Motivational interviewing: Preparing people for change. Vol. 2nd ed. Guilford Press; New York: 2002. p. 217-250.

Carty K. Testing if causal attribution is a moderator or mediator of brief treatment for problem drinkers. Dissertation Abstracts International 2005;66(3A):1161.(UMI No. 3167355)

Cherpitel CJ. Drinking patterns and problems: A comparison of ER patients in an HMO and in the general population. Alcoholism: Clinical and Experimental Research 1992;16:105-110.

Cherpitel CJ. Analysis of cut points for screening instruments for alcohol problems in the emergency room. Journal of Studies on Alcohol 1995;56:695-700. [PubMed: 8558901]

Cherpitel CJ. Alcohol in fatal and nonfatal injuries: A comparison of coroner and emergency room data from the same country. Alcoholism: Clinical and Experimental Research 1996;20:338-342.

Cisler RA, Zweben A. Development of a composite measure for assessing alcohol treatment outcome: Operationalization and validation. Alcoholism: Clinical and Experimental Research 1999;23:263271.

DiClemente CC, Marinilli AS, Singh M, Bellino LE. The role of feedback in the process of health behavior change. American Journal of Health Behavior 2001;25:217-227. [PubMed: 11322620] 
Dunn C, Deroo L, Rivara FP. The use of brief interventions adapted from motivational interviewing across behavioral domains: A systematic review. Addiction 2001;96:1725-1742. [PubMed: 11784466]

Edwards JR, Lambert LS. Methods for integrating moderation and mediation: A general analytical framework using moderated path analysis. Psychological Methods 2007;12:1-22. [PubMed: 17402809]

Emmen MJ, Schippers GM, Wollersheim H, Bleijenberg G. Adding psychologist's intervention to physicians' advice to problem drinkers in the outpatient clinic. Alcohol and Alcoholism 2005;40:219-226. [PubMed: 15699056]

Fleming MF, Barry KL, MacDonald R. The alcohol use disorders alcohol identification test (AUDIT) in a college sample. International Journal of the Addictions 1991;26:1173-1185. [PubMed: 1743817]

Heather N, Rollnick S, Bell A, Richmond R. Effects of brief counseling among male heavy drinkers identified on general hospital wards. Drug and Alcohol Review 1996;15:29-38. [PubMed: 16203349]

Hettema J, Steele J, Miller WR. Motivational interviewing. Annual Review of Clinical Psychology 2005;1:91-111.

Hungerford, DW.; Williams, JM.; Helmkamp, JC.; Furbee, PM.; Horn, K.; Presscott, JE., et al. The feasibility of screening and brief intervention for alcohol problems (SBI) among young adults in a rural, university emergency department. National Center for Injury Prevention and Control, Centers for Disease Control and Prevention, \& Center for Rural Emergency Medicine, West Virginia University; Atlanta, GA: 1999.

Kazdin AE, Nock MK. Delineating mechanisms of change in child and adolescent therapy: Methodological issues and research recommendations. Journal of Child Psychology and Psychiatry 2003;44:1116-1129. [PubMed: 14626454]

Lee CS, Longabaugh R, Baird J, Abrantes AM, Borrelli B, Stein LAR, et al. Do patient intervention ratings predict alcohol-related consequences. Addictive Behaviors 2007;32:3136-3141. [PubMed: 17720325]

Lee CS, Longabaugh R, Baird J, Nirenberg T, Mello MJ. The change plan as an active ingredient of brief motivational interventions for reducing negative consequences of drinking in hazardous drinking emergency department patients. 2009Manuscript in preparation

Longabaugh $\mathrm{R}$. The search for mechanisms of change in behavioral treatments for alcohol use disorders: A commentary. Alcoholism: Clinical and Experimental Research 2007;31(53):21S-32S.

Longabaugh R, Minugh PA, Nirenberg TD, Clifford PR, Becker B, Woolard R. Injury as a motivator to reduce drinking. Academic Emergency Medicine 1995;2:817-825. [PubMed: 7584769]

Longabaugh, R.; Wirtz, PW. Longabaugh, R.; Wirtz, PW., editors. Project MATCH hypotheses: Results and causal chain analyses. Washington DC: U.S. Government Printing Office; 2001. Substantive review and critique. NIAAA Project MATCH Monograph Series Vol. 8

Longabaugh R, Woolard RF, Nirenberg TD, Minugh PA, Becker B, Clifford P, et al. Evaluating the effects of a brief motivational intervention for injured drinkers in the emergency department. Journal of Studies on Alcohol 2001;62:806-816. [PubMed: 11838918]

MacKinnon, DP. Introduction to statistical mediation analysis. Erlbaum; Mahwah, NJ: 2008.

MacKinnon DP, Fairchild AJ, Fritz MS. Mediation analysis. Annual Review of Psychology 2007;58:593614.

MacKinnon DP, Fritz MS, Williams J, Lockwood CM. Distribution of the product confidence limits for the indirect effect: Program PRODCLIN. Behavior Research Methods 2007;39:384-389. [PubMed: 17958149]

Miller, WR.; Rollnick, S. Motivational interviewing: Preparing people to change addictive behavior. Guilford Press; New York: 1991.

Miller, WR.; Rollnick, S. Motivational interviewing: Preparing people to change addictive behavior. Vol. 2nd ed. Guilford Press; New York: 2002.

Miller, WR.; Tonigan, JS.; Longabaugh, R. The drinker inventory of consequences (DrInC): An instrument for assessing adverse consequences of alcohol abuse [test manual]. U.S. Department of Health and Human Services; Rockville, MD: 1995. NIAAA Project MATCH Monograph Series Vol. 4; NIH Publication No. 95-3911) 
Miller, WR.; Wilbourne, PL.; Hettema, JE. What works? A summary of alcohol treatment outcome research. In: Hester, RK.; Miller, WR., editors. Handbook of alcoholism treatment approaches: Effective alternatives. Vol. 3rd ed. Allyn \& Bacon; Boston, MA: 2003. p. 13-63.

Miller, WR.; Zweben, A.; DiClemente, CC.; Rychtarik, RG. Motivational enhancement therapy manual: A clinical research guide for therapists treating individuals with alcohol abuse and dependence. Washington, DC: U.S. Government Printing Office; 1992. NIAAA Project MATCH Monograph Series, Vol. 2; DHHS Publication No. ADM 92-1894

Minugh, PA.; Stein, LAR.; Longabaugh, R.; Becker, BM.; Carty, K.; Clifford, PR., et al. Immediate impact of injury as a motivator to change drinking among emergency department patients. 2009. Unpublished manuscript

Monti PM, Colby SM, Barnett NP, Spirito A, Rohsenow DJ, Myers M, et al. Brief intervention for harm reduction with alcohol-positive older adolescents in a hospital emergency department. Journal of Consulting and Clinical Psychology 1999;67:989-994. [PubMed: 10596521]

Morgenstern J, Longabaugh R. Cognitive-behavioral treatment for alcohol dependence: A review of evidence for its hypothesized mechanisms of action. Addiction 2000;95:1475-1490. [PubMed: 11070524]

Muller D, Judd CM, Yzerbyt VY. When moderation is mediated and mediation is moderated. Journal of Personality and Social Psychology 2005;89:852-863. [PubMed: 16393020]

Nirenberg, T.; Sparadeo, F.; Longabaugh, R. Brief motivational interventions for drinking injured emergency department patients. Brown Medical School, Center for Alcohol \& Addiction Studies; Providence, RI: 1996. [unpublished manual]

Nock MK, Kazdin AE. Randomized controlled trial of a brief intervention for increasing participation in parent management training. Journal of Consulting and Clinical Psychology 2005;73:872-879. [PubMed: 16287387]

Preacher KJ, Rucker DD, Hayes AF. Addressing moderated mediation hypotheses: Theory, methods, and prescriptions. Multivariate Behavioral Research 2007;42:185-227.

Prochaska JO, DiClemente CC. Stages and processes of self-change of smoking: Toward an integrative model of change. Journal of Consulting and Clinical Psychology 1983;51:390-395. [PubMed: 6863699]

Richmond R, Heather N, Wodak A, Kehoe L, Webster I. Controlled evaluation of a general practicebased brief intervention for excessive drinking. Addiction 1995;90:119-132. [PubMed: 7888970]

Rohsenow DJ, Monti PM, Martin RA, Colby SM, Myers MG, Gulliver SB, et al. Motivational enhancement and coping skills training for cocaine abusers: Effects on substance use outcomes. Addiction 2004;99:862-874. [PubMed: 15200582]

Saunders JB, Aasland OG, Babor TF, de la Fuenta JR, Grant M. Development of the Alcohol Use Disorders Identification Test (AUDIT): WHO collaborative project on early detection of persons with harmful alcohol consumption: II. Addiction 1993;88:791-804. [PubMed: 8329970]

Slavet J, Stein LAR, Colby S, Barnett N, Monti P, Golembeske C, et al. The marijuana ladder: Measuring motivation to change marijuana use in incarcerated adolescents. Drug Alcohol Dependence 2005;83:42-48.

Stotts AL, Schmitz JM, Rhoades HM, Grabowski J. Motivational interviewing with cocaine-dependent patients: A pilot study. Journal of Consulting and Clinical Psychology 2001;69:858-862. [PubMed: 11680565]

Velicer W, Redding C, Xiaowu S, Prochaska J. Demographic variables, smoking variables, and outcome across five studies. Health Psychology 2007;26:278-287. [PubMed: 17500614]

Willenbring M. A broader view of change in drinking behavior. Alcoholism: Clinical and Experimental Research 2007;31:84S-86S.

Woolard RH, Carty K, Wirtz P, Longabaugh R, Nirenberg TD, Minugh PA, et al. Research fundamentals: Follow-up of subjects in clinical trials: Addressing subject attrition. Academic Emergency Medicine 2004;11:859-866. [PubMed: 15289193] 

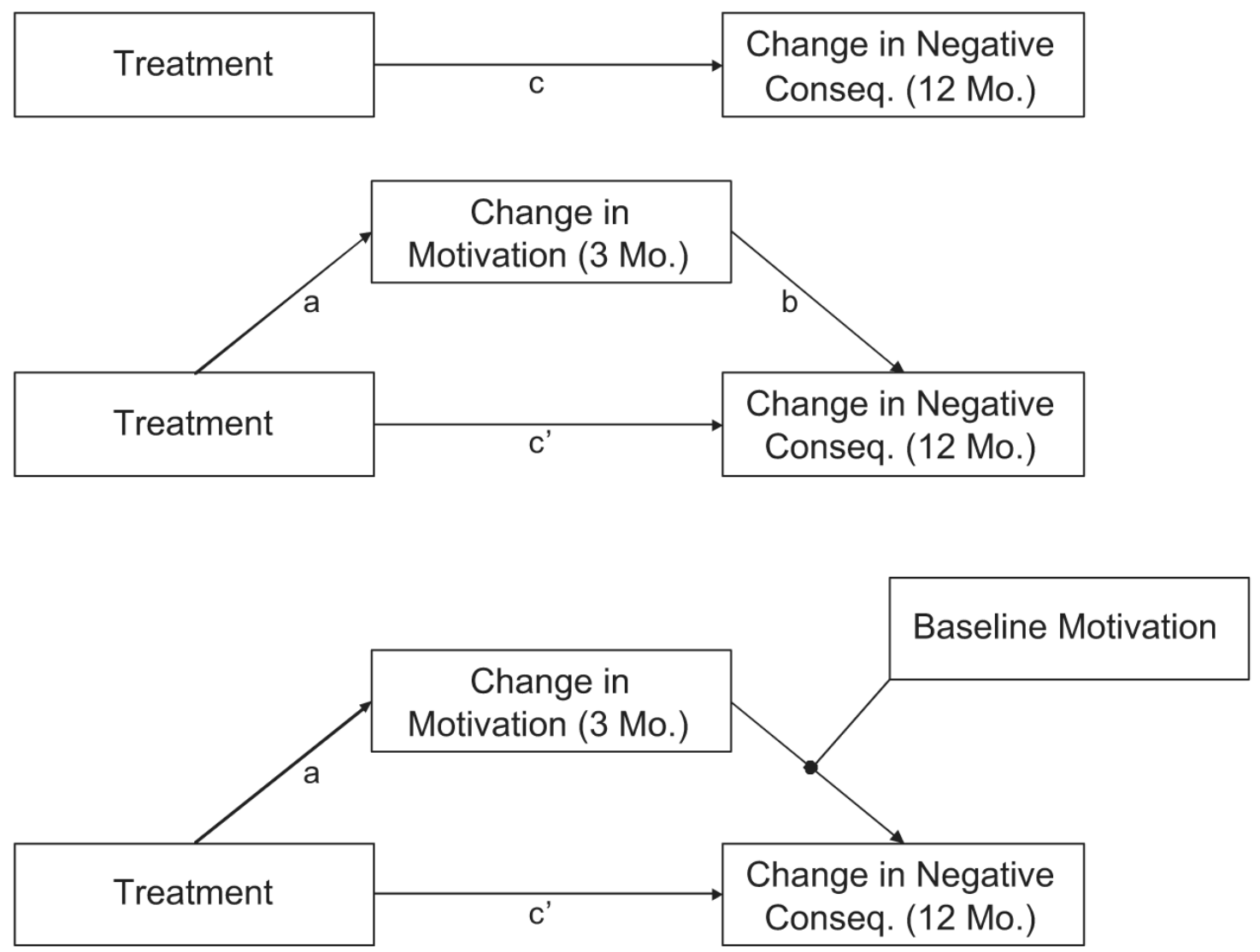

Figure 1.

Mediational model. 
Table 1

DrInC and Readiness to Change Univariate Characteristics

\begin{tabular}{lcccc}
\hline $\begin{array}{l}\text { Distributional } \\
\text { characteristic }\end{array}$ & $\begin{array}{c}\text { DrInC } \\
\text { baseline }\end{array}$ & $\begin{array}{c}\text { DrInC 12 } \\
\text { months }\end{array}$ & $\begin{array}{c}\text { Readiness to } \\
\text { change at } \\
\text { baseline }\end{array}$ & $\begin{array}{c}\text { Readiness to } \\
\text { change at 3 } \\
\text { months }\end{array}$ \\
\hline Minimum & 0.0 & 0.0 & 0.0 & 0.0 \\
25th percentile & 7.0 & 4.0 & 0.0 & 0.0 \\
Median & 13.0 & 11.0 & 1.0 & 2.0 \\
75th percentile & 22.0 & 23.0 & 3.0 & 4.0 \\
Maximum & 44.0 & 135.0 & 4.0 & 4.0 \\
Mean & 15.2 & 17.2 & 1.7 & 2.0 \\
Standard deviation & 10.6 & 19.5 & 1.5 & 1.6 \\
Standard error & 0.5 & 1.0 & 0.1 & 0.1 \\
\hline
\end{tabular}

Note. DrInC = Drinker Inventory of Consequences. Baseline DrInC covers lifetime period of time; 12 -month DrInC covers last 12 months. As the response sets for the lifetime and prior 12 months are different, the scores cannot be compared. 


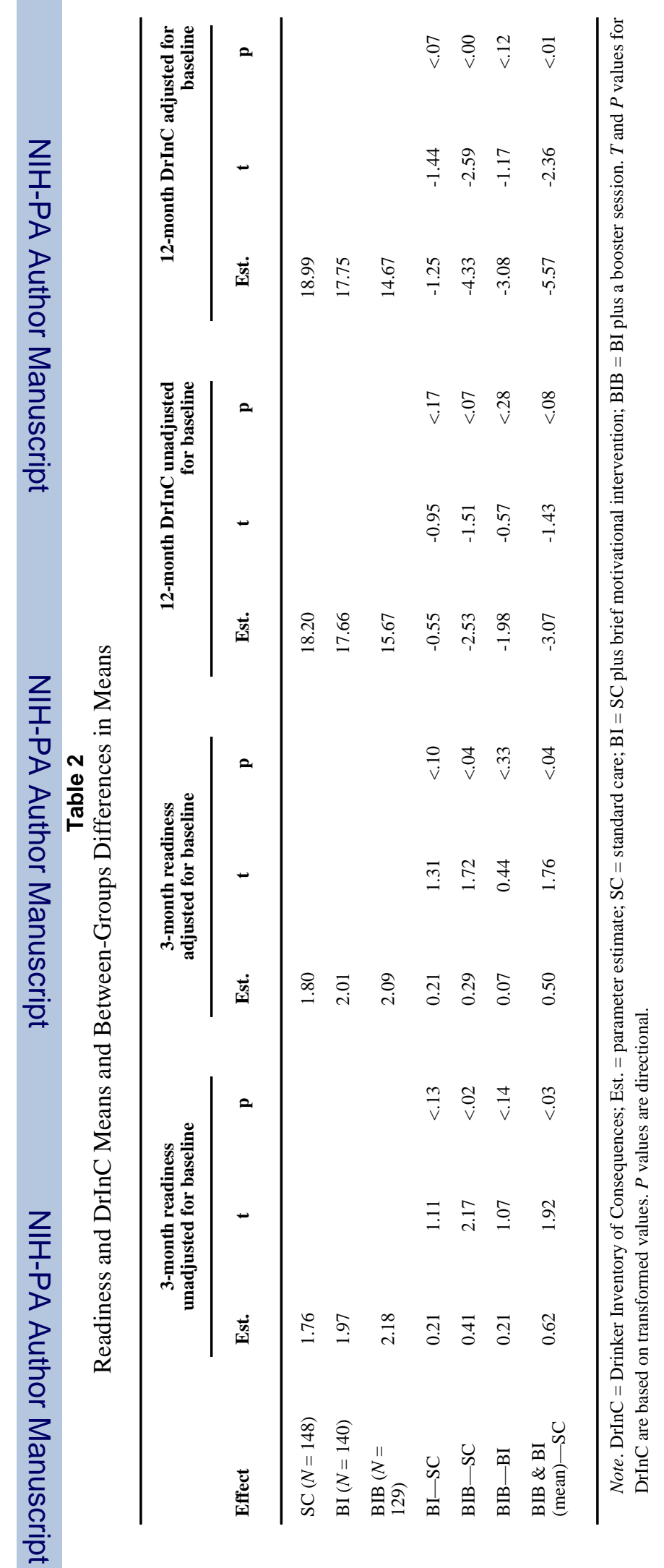


Stein et al.

Page 19

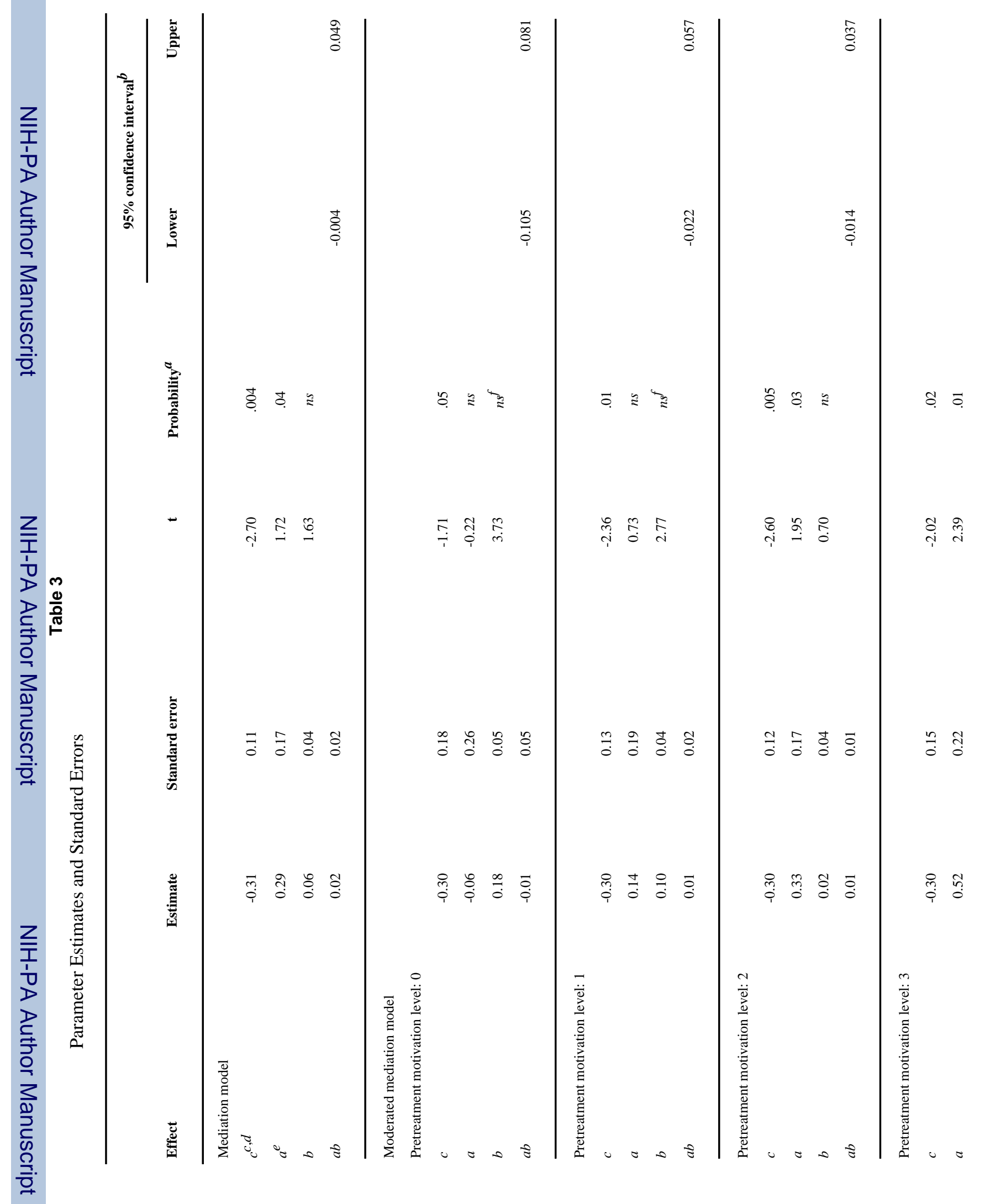


Stein et al.

Page 20

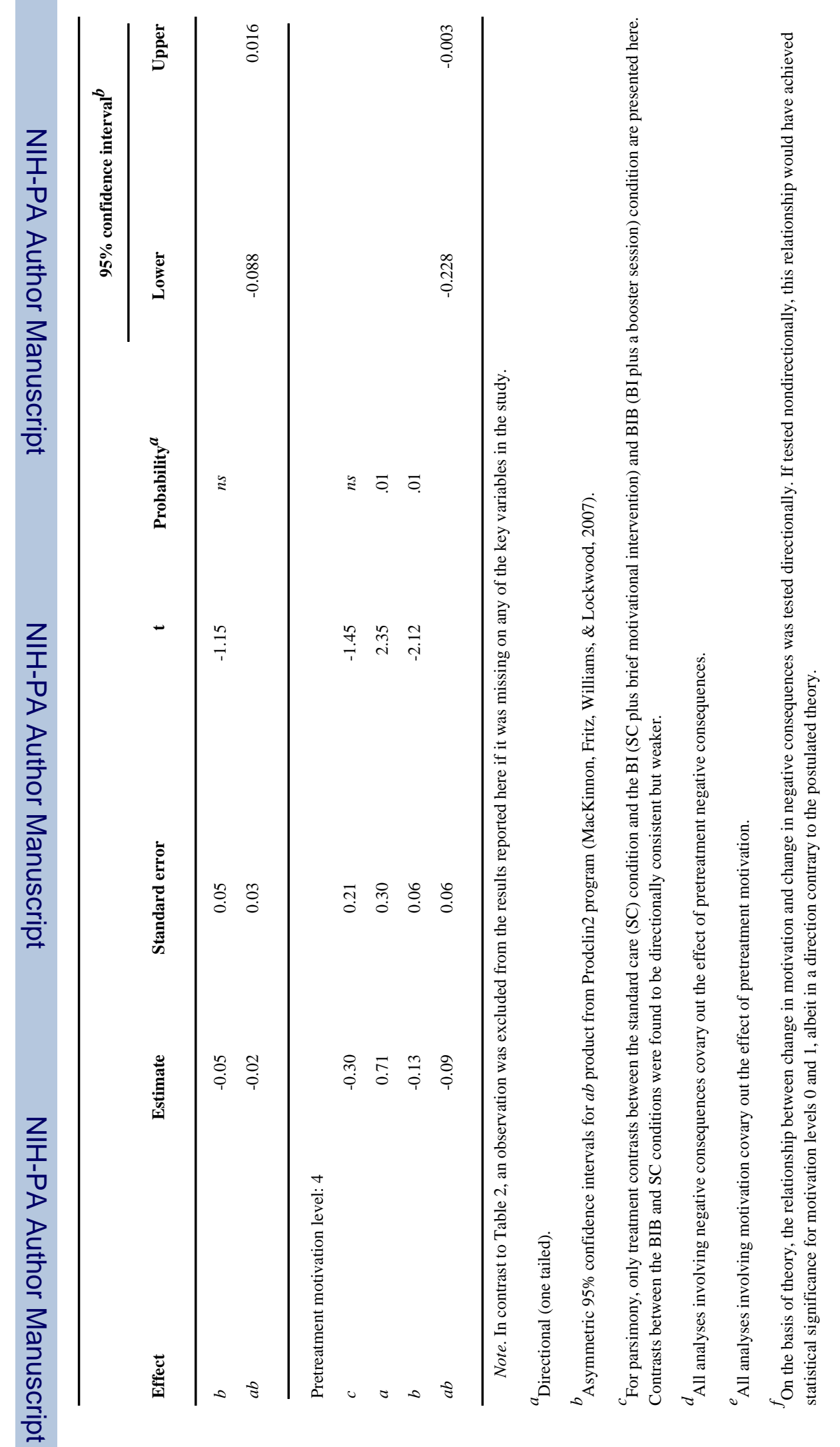

Psychol Addict Behav. Author manuscript; available in PMC 2009 September 29. 